\title{
Influence of Platelet-Rich Plasma on Chondrogenic Differentiation and Proliferation of Chondrocytes and Mesenchymal Stem Cells
}

\author{
Anja Drengk $^{\mathrm{a}}$ Antonia Zapf ${ }^{\mathrm{b}}$ Ewa Klara Stürmer ${ }^{\mathrm{a}} \quad$ Klaus Michael Stürmer $^{\mathrm{a}}$ \\ Karl-Heinz Frosch ${ }^{\text {a }}$ \\ Departments of ${ }^{\mathrm{a}}$ Trauma Surgery, Plastic and Reconstructive Surgery and ${ }^{\mathrm{b}}$ Medical Statistics, Georg August \\ University Göttingen, Göttingen, Germany
}

\section{Key Words}

Cartilage defects $\cdot$ Chondrocytes $\cdot$ Mesenchymal stem cells ·

Platelet-rich plasma $\cdot$ Reimplantation

\begin{abstract}
Background/Aims: Autologous chondrocyte (CC) transplantation has the disadvantages of requiring two surgical interventions and in vitro expansion of cells, implying the risk of cellular dedifferentiation. Our clinical aim is to develop a one-step procedure for autologous CC transplantation, i.e. harvesting, isolation and reimplantation of CC performed in one single surgical procedure. Platelet-rich plasma (PRP) is a source of autologous growth factors reported to have mitogenic effects. The objective of this study was to test the influence of PRP as an autologous scaffold on freshly isolated CC and mesenchymal stem cells (MSC). Methods: CC and MSC were subjected to two- or three-dimensional (3D) growth systems, either with or without PRP. Chondrogenic differentiation was determined via quantification of collagen type II mRNA and immunohistochemical staining. $\boldsymbol{R e}$ sults: We observed a proliferative effect for MSCs exposed to PRP in monolayer culture and an increase in the expression of chondrogenic markers when cells are exposed to a 3D environment. CCs exposed to PRP show a decrease in the chondrogenic phenotype with increasing proliferative ac-
\end{abstract}

tivity. Conclusion: PRP has a proliferative effect on CCs and MSCs. In a one-step procedure for autologous CC transplantation, this might be an advantage over other scaffold materials, but confirmation in in vivo studies is required.

Copyright $\odot 2008$ S. Karger AG, Basel

\section{Introduction}

Treatment of articular cartilage degeneration is of major concern in modern health care. Progressive loss of cartilage on joint surfaces can result in osteoarthritis [Fritz et al., 2006], which often means a painful reduction in quality of life for the patient.

Articular cartilage is known to be a tissue with limited capacity for healing spontaneously, due to lack of vascularization [Gardner, 1994]. Consequently, nutrition of the tissue depends on diffusion only. Another factor contrib-

\begin{tabular}{ll} 
Abbreviations used in this paper \\
\hline CC & chondrocyte(s) \\
MSC & mesenchymal stem cell(s) \\
PRP & platelet-rich plasma
\end{tabular}

PD Dr. Karl-Heinz Frosch

Abteilung Unfallchirurgie, Plastische und Wiederherstellungschirurgie Georg-August-Universität Göttingen

Robert-Koch-Strasse 40, DE-37075 Göttingen (Germany)

Tel. +49 551398 989, Fax +49 551398 991, E-Mail khfrosch@med.uni-goettingen.de 
uting to the poor regenerative capacity of articular cartilage is the fact that it is a tissue with a restricted number of cells. The percentage of highly specialized chondrocytes (CC), which are embedded within the matrix rich in collagen fibrils and proteoglycans, is only $1-3 \%$ [Fritz et al., 2006]. CC embedded in extracellular matrix are unable to migrate to a site of injury [Buckwalter and Mankin, 1998 ] and, therefore, only defects $<3 \mathrm{~mm}$ in diameter can be self-repaired [Kim et al., 1991].

Some well-established treatment procedures with good initial outcome with respect to pain relief and mobility do exist. However, these treatments are far from satisfactory as they mostly result in the formation of biomechanically inferior fibrocartilage, which in the long term does not prevent the development of osteoarthritis. Procedures such as debridement [Magnuson, 1974], abrasion arthroplasty [Johnson, 2001], drilling [Pridie, 1959], microfracturing [Steadman et al., 2001] and autologous CC transplantation are available, with the procedure of choice dependent on the depth and width of the defect. For autologous CC transplantation, a piece of cartilage must be harvested from the non-weight-bearing site of the joint. CC are then isolated and expanded under good medical practice conditions and, finally, reimplantated at the site of cartilage injury, usually embedded in a collagen fleece. The defect is then closed with a periosteal flap. This procedure suggests promising results [Niethard and Schneider, 2004] but has some disadvantages. First, two surgical interventions are required and secondly, CC expansion is a slow process and therefore bears several risks, e.g. CC dedifferentiation and contamination.

Our clinical goal was to develop a one-step procedure, allowing CC harvesting, isolation and reimplantation to be performed in a single surgical intervention. However, applying this method, there would obviously be no increase in the number of CC. The additional use of platelet-rich plasma (PRP), which has been shown to have a mitogenic effect on different cell types in vitro [Gruber et al., 2002; Weibrich et al., 2002; Arpornmaeklong et al., 2004; Gruber et al., 2004; Choi et al., 2005], could be utilized to increase the cell number. PRP is a source of autologous cytokines, chemokines and growth factors which, in the case of injury, manage and control the process of wound healing and tissue repair. The most prominent growth factors in PRP are platelet-derived growth factor (PDGF), transforming growth factor (TGF)- $\beta_{1}$ and TGF- $\beta_{2}$, epidermal growth factor, insulin-like growth factor, basic fibroblast growth factor and vascular endothelial growth factor, which influence cell proliferation and differentiation [Anitua et al., 2004]. The aim of this study was, therefore, to test the influence of PRP on proliferation and differentiation of freshly isolated CC and mesenchymal stem cells (MSC) of sheep in an in vitro study.

\section{Materials and Methods}

Isolation and Cultivation of CC and MSC

Pieces of cartilage from the femoral condyle of an adult female sheep were collected. CC were isolated via overnight digestion with collagenase type II from PAN (Biotech, Germany), with a final enzyme concentration of $2 \mathrm{mg} / \mathrm{ml}$. Subsequently, cells were passaged through a $70-\mu \mathrm{m}$ cell strainer (Falcon; BD Bioscience, Erembodegem, Belgium) to remove any undigested cartilage, washed twice with DMEM (Gibco, Germany) and counted. Cell viability was checked with the trypan blue exclusion test.

MSC were obtained using a standardized modified protocol according to Pittenger [1999]. With an 18-gauge syringe, bone marrow was aspirated under anesthesia from the iliac crest of the sheep mentioned above. Cells isolated by density gradient centrifugation were plated in DMEM supplemented with 10\% FCS, $2 \mathrm{mmol} / \mathrm{l} \mathrm{L}$-glutamine and $50 \mu \mathrm{g} / \mathrm{ml}$ penicillin-streptomycin. Culture medium was changed twice a week. Cells formed symmetric colonies within 10-14 days and reached confluence after 3-4 weeks. Cells were used for assays after the second passage. Under specific culture conditions [Pittenger et al., 1999], cells obtained as described were able to differentiate into a chondrogenic and osteoblastic lineage.

In all experimental approaches, the culture medium was the same for all cell types, DMEM supplemented with $10 \%$ FCS, $2 \mathrm{mmol} / \mathrm{l} \mathrm{L}$-glutamine, $50 \mu \mathrm{g} / \mathrm{ml}$ ascorbic acid and $50 \mu \mathrm{g} / \mathrm{ml}$ penicillin-streptomycin. Cells were cultured at $37^{\circ} \mathrm{C}$ and $5 \% \mathrm{CO}_{2}$.

\section{Preparation of $P R P$}

To produce PRP extracts, blood was collected from the ear vein of the anesthetized sheep into $7.5-\mathrm{ml}$ monovettes supplemented with sodium citrate $(3.8 \%)$ at a ratio of 9 volumes blood and 1 volume sodium citrate according to Anitua [2004]. The citrated blood was centrifuged in a standard laboratory centrifuge (Labofuge 400; Heraeus) for $10 \mathrm{~min}$ at 2,400 rpm. Subsequently, the yellow plasma from 6 monovettes was transferred to a $50-\mathrm{ml}$ plastic tube and platelets were pelleted in a second centrifugation step for $15 \mathrm{~min}$ at 3,600 rpm (protocol according to Weibrich et al. [2001], slightly modified). The pellet was resuspended in 1,200 $\mu l$ of plasma and the platelets counted in a Neubauer counting chamber. The PRP was stored at $4^{\circ} \mathrm{C}$ until the next day when the isolated CC were ready for use. Thus, in the experimental setup, CC received autologous PRP preparations, whereas MSC were treated with homologous PRP preparations. PRP $(150 \mu \mathrm{l})$ was activated with $7.5 \mu \mathrm{l} \mathrm{CaCl}_{2}(10 \%)$ in a $1.5-\mathrm{ml}$ reaction tube, mixed and either left until clotted, mixed with cells and carefully placed into a culture well in the form of a drop, or pipetted onto the bottom of a cell culture flask as a thin fibrin gel smear.

\section{Treatment Groups}

We had six different treatment groups, each starting with $4 \times$ $10^{5}$ cells: (1) cultivation of cells in a high-density, three-dimensional (3D) pellet culture system; (2) same as (1) but with the ad- 
dition of activated PRP; (3) cells suspended in a fibrin sealant clot; (4) cells suspended in a PRP clot; (5) cells growing in monolayer, and (6) same as (5) but with the addition of activated PRP (fig. 1).

To produce the $3 \mathrm{D}$ pellet (treatment group 1), cells were suspended in $2.5 \mathrm{ml}$ of medium in a $15-\mathrm{ml}$ plastic tube and centrifuged for $5 \mathrm{~min}$ at $500 \mathrm{~g}$, according to Tallheden et al. [2004]. The pelleted cells aggregated and formed a stable micromass. For treatment group 2, $150 \mu \mathrm{l}$ of PRP was activated as described above and the resulting clot added to the culture medium, where it was kept buoyed up on the surface of the medium. In group 3, we employed a fibrin sealant set (Tissucol; Baxter, Heidelberg, Germany), preparing the solutions according to the manufacturer's instructions but using a final dilution of the stock solutions of $1: 2.4 \times 10^{5}$ cells were resuspended in $75 \mu$ l of the fibrinogen component and mixed with $75 \mu$ l of the thrombin solution. The resulting cell fibrin-sealant construct was cultured in a 6 -well culture dish. For treatment group 4, the same amount of cells was resuspended in $150 \mu \mathrm{l}$ PRP and activation was carried out as described above. The resulting cell-PRP clot was cultured analogous to group 3. Treatment groups 5 and 6 comprised cells grown as a monolayer in 75-ml culture flasks either in the absence (5) or in the presence of PRP (6).

Each individual cell isolate was submitted to all of the six treatment groups and each group (except for 5 and 6) was prepared twice, once for immunohistochemical analysis and once for RNA preparation. Culture time was 14 days. At the end of culture, cells grown in monolayer were harvested as described previously [Frosch et al., 2003] and counted in a Coulter counter.

\section{Histochemical and Immunohistochemical Procedures}

After culture, micromasses and cell-PRP/cell fibrin-sealant clots were fixed in $4 \%$ paraformaldehyde overnight, dehydrated in increasing concentrations of ethanol and embedded in paraffin. Three-micrometer sections were cut and placed on microscope slides, deparaffinized and stained either with toluidine blue or immunoassayed with an antibody against collagen type II (mouse anti-chicken collagen, monoclonal; Dunn Labortechnik, Germany). Sections that underwent staining for collagen type II were pretreated with proteolytic enzyme (Dako, Germany) for 10 min at $37^{\circ} \mathrm{C}$ to improve accessibility of the antibody and to expose epitopes that may have been masked during fixation. All sections were incubated with $3 \% \mathrm{H}_{2} \mathrm{O}_{2}$ for $10 \mathrm{~min}$ at room temperature to eliminate endogenous peroxidase activity and subsequently received a protein block (Dako). Incubation with primary antibody was carried out overnight for anti-collagen type II (dilution 1:50). The secondary horseradish-peroxidase-conjugated antibody (goat anti-mouse; Dako) was incubated for $1 \mathrm{~h}$ and, after rinsing, sections were developed with 3-amino-9-ethyl carbazole (Sigma, Deisenhofen, Germany) for 30 min, counterstained with hemalum (Merck, Darmstadt, Germany) and mounted in Aquamount (BDH Laboratory Supplies, Poole, UK). The stained sections were analyzed with a Leica DM IRBE microscope.

\section{Quantitative Real-Time PCR}

For total RNA preparation, we used a RNA/DNA Kit (Qiagen, Germany). Cells grown in monolayer, micromasses, cell-PRP and cell fibrin-sealant clots were harvested and transferred into lysis buffer, homogenized through an 18-gauge needle and processed following the manufacturer's instructions. After samples had been treated with DNase to remove possible contamination of genomic DNA, first-strand complementary DNA was synthesized from 400 ng total RNA using specific reverse primers for GAPDH and collagen type II using Superscript II Reverse Transcriptase (Invitrogen, Karlsruhe, Germany). From this reaction, $2.5 \mu$ l were used in a SYBR-Green PCR reaction (QuantiTect SYBR-Green PCR Kit; Qiagen) run in the iCyler iQ Real-Time Detection System (Bio-Rad, Germany). The mRNA expression level of the target gene in each treatment group was determined relative to the expression level of the target gene of the calibrator control group 5 ; each value was normalized to the reference gene GAPDH and calculated according to the Pfaffl method for different amplification efficiencies (Bio-Rad Laboratories, Real-time PCR Application Guide). Quality of the PCR products was checked by melting curve analysis and electrophoresis of the PCR product. Primer sequences were as follows: KIIE3fw: $5^{\prime}$-GAAACCAGGACCAAAGGG-3'; KIIE3rv: 5'-CTTGTTCCCCTGCAGGTC-3'; GAPDHovisfw: 5'-CCTTCATTGACCTTCACTACATGGTCT3', and GAPDHovisrv: 5'-TGGAAGATGGTGATGGCCTTTCCATTG-3'. The primers for GAPDH were according to Bosnakovski et al. [2004]. The identity of each PCR product was confirmed by sequence analysis.

\section{Statistical Evaluation}

Data were analyzed using SAS 9.1 software (SAS Institute, Cary, N.C., USA). To investigate the effect of the different treatments, we performed a mixed linear model analysis. Pairwise comparisons between treatment groups were performed by Tukey's procedure.

\section{Results}

\section{Influence of PRP on Cell Proliferation}

At the end of the experiment, cells grown in monolayers either in the absence (treatment group 5) or in the presence of PRP (treatment group 6) were trypsinized and counted. For MSC, there were 8 individual and independent cell isolates. In 7 of 8 cases, a higher cell count was observed for samples grown in the presence of PRP (fig. 2a). For CC, the influence on cell proliferation was not as homogeneous as for MSCs. In 6 of 9 cases, cells grown in the presence of PRP showed an increase in cell number compared to those without PRP supplement, indicating a positive influence of PRP on cell proliferation (fig. 2). Based on the starting cell number, there was an average increase of about 14-fold for MSC at the end of the experiment compared to an average increase of about 10 -fold for CCs.

Influence of PRP on Immunohistochemical Evaluation

None of the MSC samples in either treatment group stained positively for collagen type II. However, quantitative real-time PCR analysis showed a weak collagen 


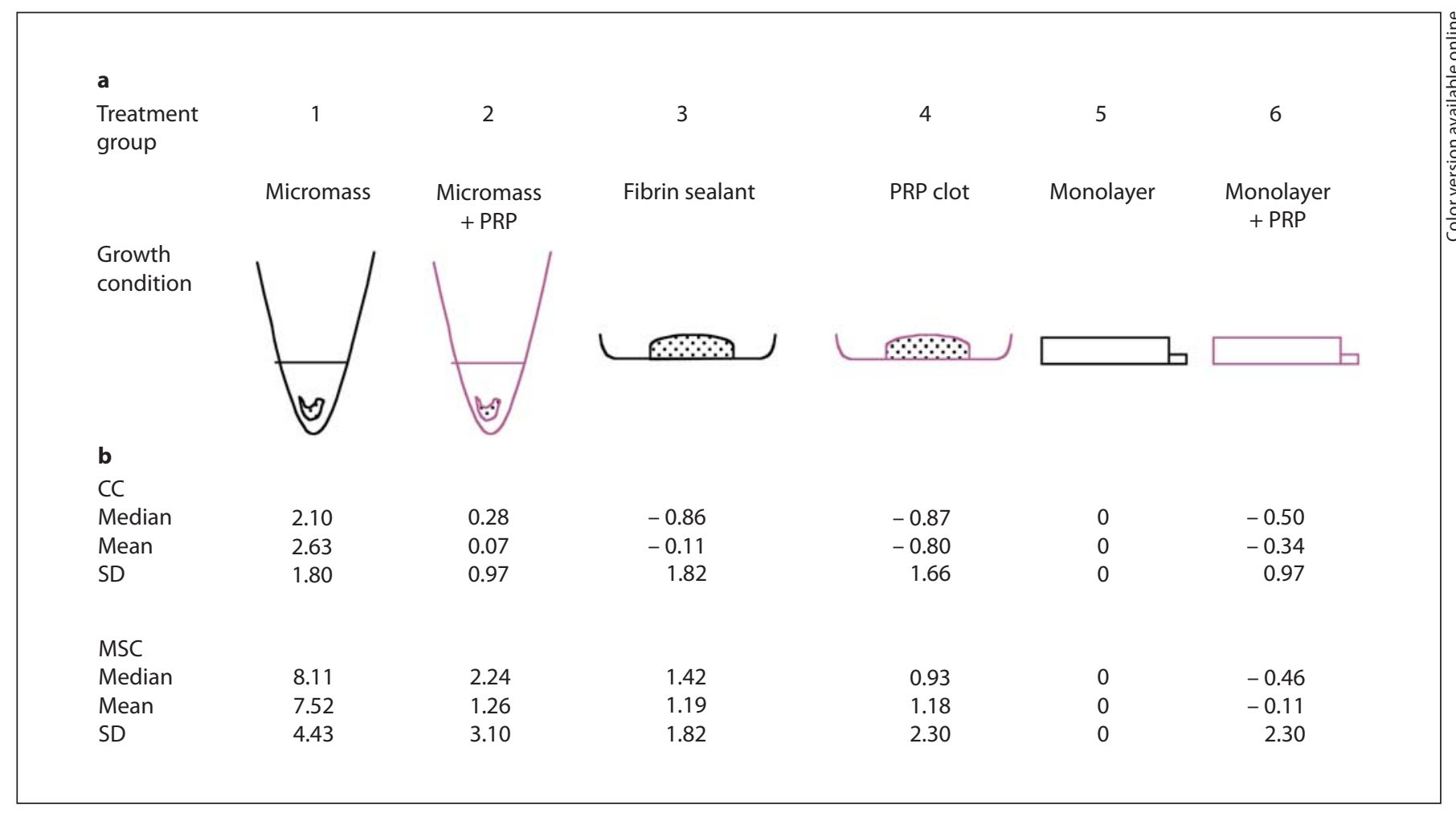

Fig. 1. a Schematic illustration of the experimental setup showing the different treatment groups and culture conditions. b Data describing the quantitative analysis of chondrogenic differentiation (level of collagen type II mRNA expression rate) of each treatment group, with treatment group 5 as calibrator control group.
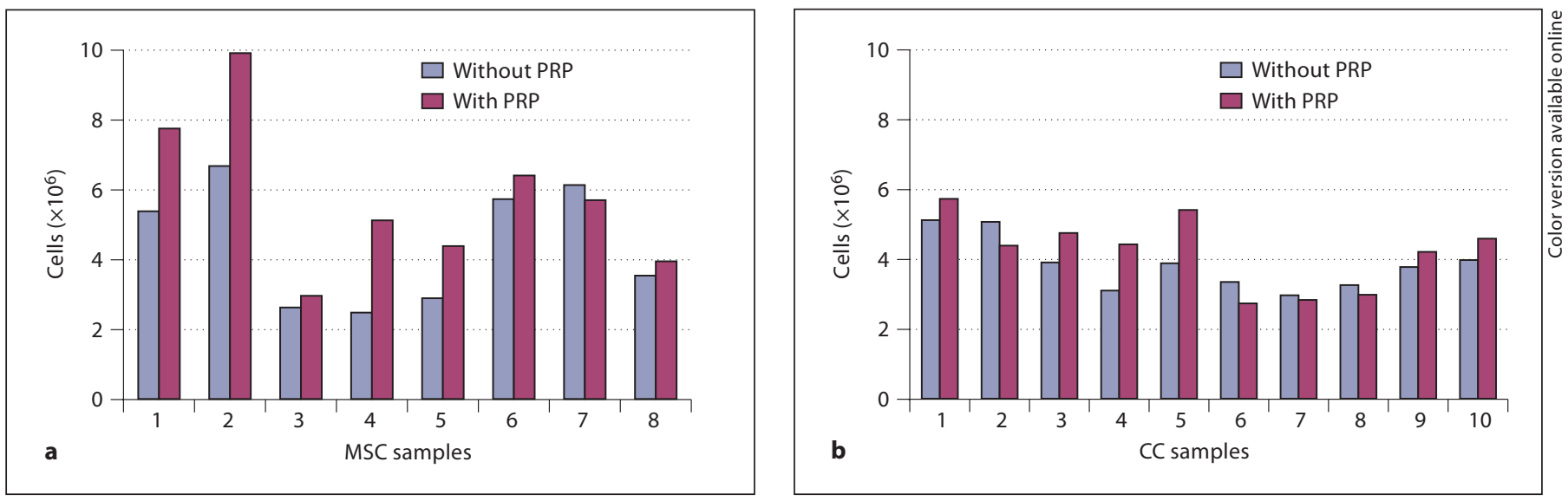

Fig. 2. Cell counts of MSC (a) and CC (b) grown in monolayer for 2 weeks either without or with PRP.

type II transcription activity, which was not sufficient for immunohistochemical detection of protein.

In contrast to the MSC samples, CC grown in micromass culture (treatment groups 1 and 2) showed distinct staining for collagen type II protein (fig. 3). As demonstrated by immunohistochemical staining, collagen type II expression is negatively influenced by the addition of PRP (treatment group 2). Staining intensity diminished 

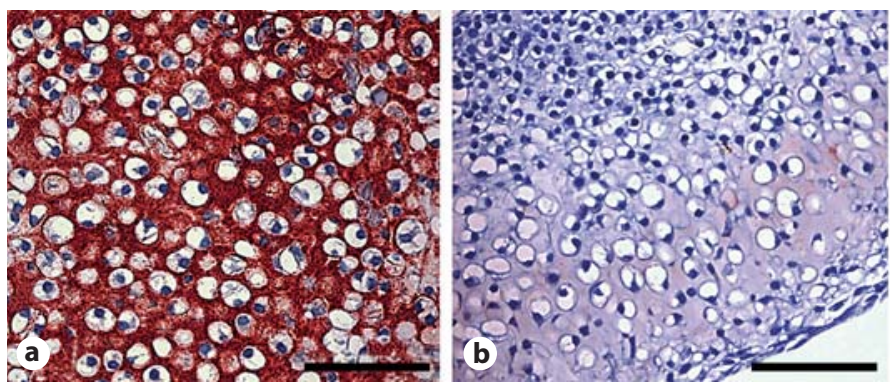

Fig. 3. Representative pictures of the immunohistochemical analysis of collagen type II expression in groups 1-4. Scale bars = $100 \mu \mathrm{m}$. Note that cells grown in micromasses $(\mathbf{a}, \mathbf{b})$ show a chondrogenic phenotype with cells sitting in lacunae being surrounded by a matrix produced entirely by the cells. This matrix stains
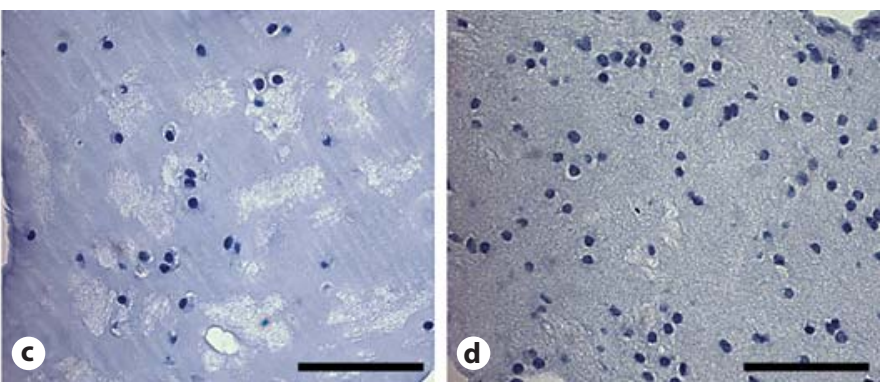

strong for collagen type II protein in treatment group 1 and weak in treatment group 2. Cells grown in a fibrin sealant (c) or PRP clot (d) have only small lacunae or none at all and there is no collagen type II staining in the surrounding scaffold.

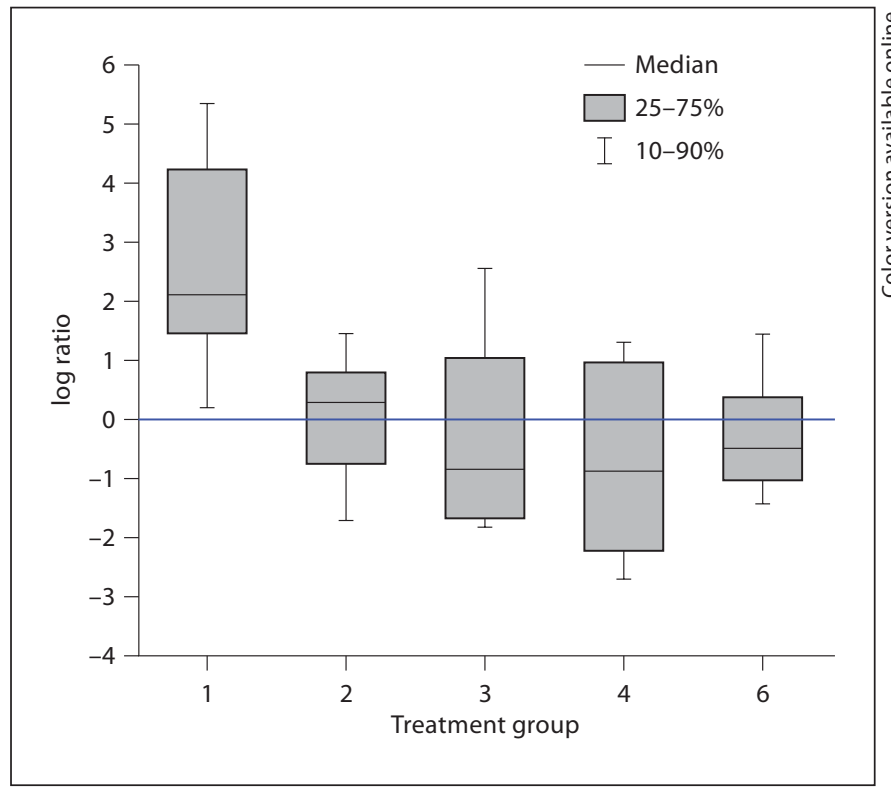

Fig. 4. Box plot illustrating the collagen type II mRNA expression level of CC for each treatment group in relation to the expression level of calibrator control group 5 (zero line). Each treatment group comprised 10 independent cell isolates. ( micromass culture dramatically reduced collagen type II mRNA expression, however the median was still above the control level and this culture condition appears superior to growing cells in a fibrin sealant clot, PRP clot, or in monolayer with or without additional PRP (fig. 1b, 4). Categories 3, 4 and 6 show a reduced median collagen type II mRNA expression level in comparison to the control group, but the difference was not significant ( $\mathrm{p}>$ 0.05).

For MSC, the results are as follows: the highest expression level of collagen type II mRNA was found in treat- 


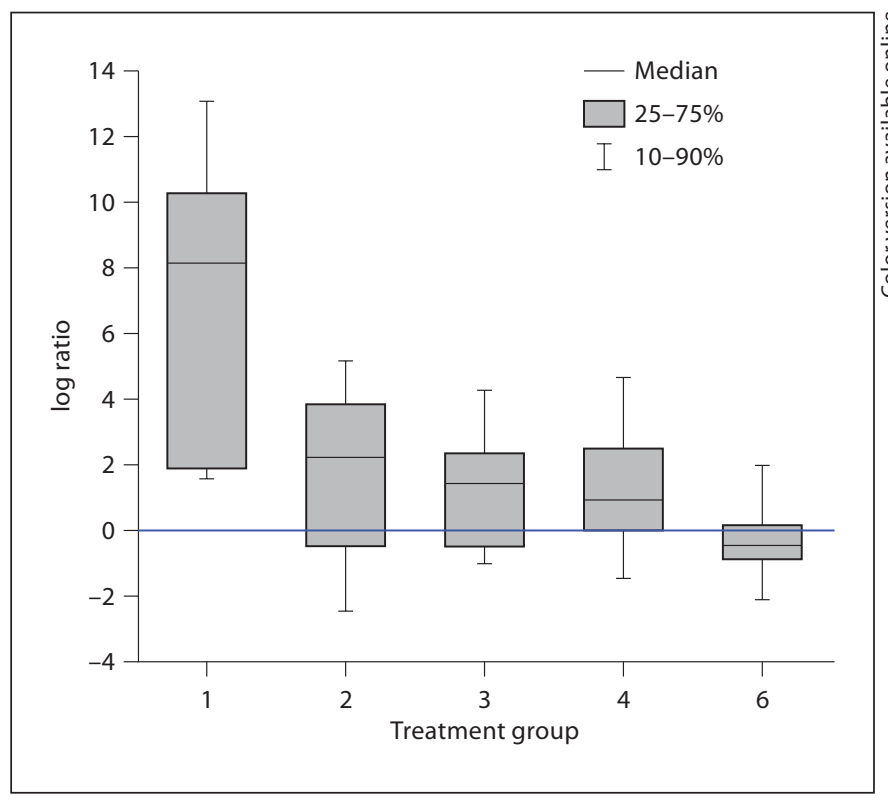

Fig. 5. Box plot illustrating the collagen type II mRNA expression level of MSC for each treatment group in relation to the expression level of calibrator control group 5 (zero line). Each treatment group comprised 8 independent cell isolates.

sults were not significant ( $p>0.05)$. Addition of PRP to the monolayer (treatment group 6) resulted in a mean collagen type II mRNA expression level below the control level.

\section{Discussion}

In recent decades, degenerative diseases of the articular knee joint have been gaining increasing clinical importance. A changed leisure behavior paralleled by an increase in the elderly population has led to a higher prevalence of articular cartilage defects, which, when untreated, often result in osteoarthritis. Treatment of such defects has evolved tremendously in recent years. PRP is currently being highly investigated, particularly in oral and maxillofacial surgery. The idea is that via application of PRP the patient's own growth factors are used to enhance healing. Marx et al. [1998] report that the addition of PRP to an autogenous cancellous bone graft results in a faster maturation rate and higher bone formation rate in alveolar defects. There are reports on the potency of PRP to improve the cohesion of an autogenous bone graft by forming a fibrin gel and to release factors leading to enhanced bone regeneration [Fuerst et al.,
2003; Fennis et al., 2004]. Anitua et al. [2004] described the stabilizing effect of PRP clots around implants in solid bone, virtually eliminating the risk of implant failure. In addition, PRP has a strong stimulant effect on capillary regeneration in wound healing [Lindeboom et al., 2007] and a neurotrophic effect in studies concerning peripheral nerve recovery [Farrag et al., 2007]. Implementation of a PRP collagen scaffold has been reported to stimulate healing of anterior cruciate ligament defects both histologically and biomechanically [Murray et al., 2006], and accelerated articular cartilage healing has been demonstrated by the use of a PRP preparation in a case of articular cartilage avulsion [Sanchez et al., 2003].

Considering all the positive effects described for PRP in various clinical applications, we aimed to establish whether the combination of MSC with PRP, or the combination of freshly isolated CC with PRP, would form a bioactive composite suited for healing of cartilage defects. This work was undertaken as a basic in vitro study to better assess the possible outcome of an in vivo experiment. For the in vitro study we added FCS to the culture medium to enhance the growth of CCs and MSCs. However, this additive would not be practical in an in vivo setting because of the risk of viral or prion-related disease transmission and foreign protein contamination. Autologous serum could be an adequate substitute for FCS, although, in the one-step procedure we propose, the cell culture step would be omitted.

PRP contains a natural cocktail of growth factors which act in concert to stop bleeding and initiate healing and restoration of damaged tissue during injury. PDGF and TGF- $\beta$ are the most prominent of these growth factors and the mitogenic effect of TGF- $\beta$ on osteoblasts and stem cells has been demonstrated [Marx et al., 1998]. Numerous in vitro studies demonstrate a mitogenic effect of PRP on various cell types, for example, on human trabecular-bone-derived cells [Gruber et al., 2002], human SaOS-2 osteoblasts [Celotti et al., 2006], human oral fibroblasts and osteoblasts [Graziani et al., 2006] and human foreskin fibroblasts [Lanas et al., 1994]. There are also several reports of mitogenic stimulation of human MSC by platelet releasates [Gruber et al., 2004; Vogel et al., 2006].

In this study, we also observed a mitogenic effect of PRP on marrow-derived stem cells from adult sheep. However, the effect was small, possibly due to the small amount of PRP used, suggesting that the amount of added growth factors acting on the monolayer cells may have been minimal. It might also be that a primary, stronger stimulating effect exerted by adding PRP was masked in 
the course of the experiment, as the most notable effect of PRP on cell proliferation has been reported to occur after $72 \mathrm{~h}$ of stimulation [Graziani et al., 2006].

There are only a few studies reporting a proliferative effect of PRP on CC [Kaps et al., 2002; Gaissmaier et al., 2005; Akeda et al., 2006], but none of these investigations stimulated freshly isolated CC with PRP. In our study, PRP exerted a proliferative effect on freshly isolated, completely differentiated CC submitted to growth in monolayer culture. Proliferation increased in $67 \%$ of CC samples, being a less homogeneous response to PRP stimulation than that observed for MSC, in agreement with results in bovine articular CC exposed to human platelet supernatant [Kaps et al., 2002], where only $40 \%$ of platelet batches displayed growth-promoting activity, an observation explained with the cell differentiation status and donor age [Blanco et al., 1995; Guerne et al., 1995].

Another reason for the variable response of cells to stimulation with PRP may be associated with the PRP preparations utilized. There are several reported procedures for the generation of PRP concentrates [Weibrich et al., 2001; Dugrillon et al., 2002; Sanchez et al., 2003; Kitoh et al., 2004; Yazawa et al., 2004; Anitua et al., 2006] and a lack of standardization for self-concentrated PRP preparations. Platelet counts differ significantly according to the preparation method. Weibrich et al. [2001] observed no significant correlation between the platelet count of self-concentrated PRP and donor whole blood. Further, 10\% of self-concentrated PRP counts were reported as lower than those of the donor whole blood and there is an additional influence of sex on platelet concentration and an interindividual variability [Weibrich et al., 2001]. A dose dependence of the PRP action has been reported previously. Choi et al. [2005] described suppression of cell viability and proliferation by high PRP concentrations for alveolar bone cells, and Graziani et al. [2006] has shown that high PRP concentrations result in a reduction in cell proliferation of human oral fibroblasts and osteoblasts. Better results for the proliferation of human FOB19 cells were obtained when PRP preparations were diluted [Ferreira et al., 2005]. Therefore, the optimal PRP concentrations remain to be determined.

We tend to see the non-uniform response of the CC samples in the current study in light of this issue. PRP preparations were not equalized in order to maintain the experimental setting as close as possible to the clinical application. PRP variability is an uncontrollable parameter in an autologous setting, similar to the donor-dependent variability of growth and differentiation competence of MSC and CC.

Influence of PRP on Chondrocytes and Mesenchymal Stem Cells
Our results show that cultivation of freshly isolated CC in a high-density micromass culture best preserves the chondrogenic phenotype compared to all other cultivation methods. Addition of PRP dramatically reduces collagen type II mRNA expression in categories 2, 4 and 6 . This is not surprising considering the positive influence of PRP on cell proliferation discussed previously. Proliferation and differentiation cannot occur in a cell simultaneously, so an increase in proliferation obviously negatively influences the differentiation status. Addition of TGF- $\beta$ decreases expression of type II mRNA in freshly isolated CC cultured in monolayer [van der Kraan et al., 1992; Fortier et al., 1998], and this observation supports our findings, as TGF- $\beta$ is one of the most prominent growth factors enriched in platelet concentrations. PDGF as the other dominant growth factor in platelet concentrates also stimulates a decrease in the amount of cartilage proteoglycan, exerting a mitogenic effect [Weiser et al., 1999]. In addition, an inhibition of cartilage differentiation in vitro by platelet supernatant has been reported previously [Kaps et al., 2002; Gaissmaier et al., 2005]. However, these studies utilized CC previously expanded in monolayer culture. We show, for the first time, that PRP also leads to a reduction in collagen type II mRNA expression in freshly isolated CC. We assume that this dedifferentiating influence is even stronger on freshly isolated CC than on cells previously expanded in monolayer. This assumption is supported by the observation that a significant difference in the level of collagen type II mRNA expression is no longer obtained between treatment groups 1 and 2 in cells previously expanded in monolayer culture [unpubl. data with rabbit CC].

In our study, a negative influence of PRP on collagen type II mRNA expression was observed in freshly isolated CC in all treatment groups, but there are clear differences in the degree of impact that PRP exerts on collagen type II mRNA expression in treatment groups 2, 4 and 6 . As an explanation we postulate two opposite forces acting on the cells. One is the above-mentioned dedifferentiating activity of PRP. The other is the $3 \mathrm{D}$ arrangement of cells, which is unique to each treatment group. In treatment group 2, cells have close cell-to-cell contact, which is a condition possibly well suited for preserving the chondrogenic phenotype. There are reports about close cell-cell contact reversing the loss of phenotype of cells grown in monolayer culture [Tallheden et al., 2004] or rather preventing such a phenotype loss [Kuettner et al., 1982; Bassleer et al., 1986]. Consistently, in this treatment group, the dedifferentiating influence of PRP is the

Cells Tissues Organs 2009;189:317-326 
weakest, whereas it is logically strongest when cells are grown in monolayer (treatment group 6), a condition known to promote dedifferentiation [von der Mark et al., 1977; Benya et al., 1978; Marlovits et al., 2004]. Treatment group 4 lies between these extremes, featuring 3D arrangement, but not close cell-to-cell contact. In treatment group 3, dedifferentiation was comparable to group 4 . With regard to the $3 \mathrm{D}$ arrangement, these two groups are comparable. There are reports describing a growth-promoting activity inherent in fibrin(ogen) [Gray et al., 1993] as well as in thrombin [Zangi et al., 2006]. The decrease in collagen type II mRNA expression in treatment group 3 might therefore be due to an increase in proliferation. Concerning the collagen type II mRNA expression, culturing freshly isolated CC in a $3 \mathrm{D}$ arrangement using fibrin sealant or PRP seems to be inferior to a simple monolayer culture. The reason for this lies in the proliferative effect of the supplements discussed above, which result in an accelerated dedifferentiation process when compared to cells grown in monolayer. For CC previously expanded in monolayer, this seems to be different [Gaissmaier et al., 2005].

For assays carried out with MSCs, culturing cells in high-density micromasses yields the highest level of collagen type II mRNA expression compared with cells grown in monolayer. It is not clear whether this is due to an increase in collagen type II expression induced through the condensed and 3D growth of the cells or whether it is the result of a better conservation of a basal collagen type II mRNA expression previously described by different authors [Majumdar et al., 2000; Worster et al., 2000]. However, several published observations seem to support the assumption that the culturing condition has led to the observed increase in collagen type II mRNA expression. Bosnakovski et al. [2004] reported that, using fetal bovine MSCs, the condensed culturing condition was a sufficient external stimulus for the cells to undergo chondrogenesis. The high cell density, which allows cellto-cell interactions analogous to those occurring in mesenchymal condensation during limb development before cartilage formation, seems to be a specific stimulus supporting chondrogenesis of MSCs from most species [Bosnakovski et al., 2004].

To our knowledge, this is the first study on the effect of PRP on chondrogenic differentiation of MSCs, and a negative effect of PRP on the level of collagen type II mRNA expression was found. Addition of PRP to a micromass culture led to a significant reduction in collagen type II mRNA. Furthermore, the addition of PRP to the monolayer culture decreased median collagen type II
mRNA expression compared to the untreated monolayer, which can be explained by the proliferation-stimulating effect of PRP. However, in treatment groups 1-4, the median expression level of collagen type II mRNA was above the control level. Only in treatment group 6 dropped the median level of collagen type II mRNA expression below the control level. In contrast to experiments carried out with CC, it can be stressed that with regard to the chondrogenic differentiation, growing MSCs in a 3D culture system is clearly superior to monolayer culture. Growing cells in a fibrin sealant clot, a PRP gel or as a micromass with or without PRP exposure results in a higher collagen type II mRNA expression level compared with monolayer culture.

Our results from sheep MSC and PRP show a clear proliferative effect for MSC exposed to PRP in monolayer culture and an increase in the expression of the chondrogenic marker collagen type II mRNA when cells are exposed to PRP in a $3 \mathrm{D}$ environment. Our results indicate that MSC in combination with PRP are able to improve the healing of articular cartilage defects in vivo.

Freshly isolated sheep CC in combination with a PRP preparation lose their chondrogenic phenotype during in vitro culture. This loss of chondrogenicity seems to be connected with an increase in proliferation. Based on these observations, it remains difficult to evaluate how the cells would develop in an in vivo environment, where they would be exposed to the adequate chondrogenic stimuli. This remains to be shown in an animal study.

\section{Acknowledgments}

The authors express their thanks to R. Castro, F. Kauer and Dr. P. Krause for experimental assistance.

References Akeda, K., H.S. An, M. Okuma, M. Attawia, K.
Miyamoto, E.J. Thonar, M.E. Lenz, R.L. Sah,
K. Masuda (2006) Platelet-rich plasma stim-
ulates porcine articular chondrocyte prolif-
eration and matrix biosynthesis. Osteoar-
thritis Cartilage 14: 1272-1280.
Anitua, E., I. Andia, B. Ardanza, P. Nurden, A.T.
Nurden (2004) Autologous platelets as a
source of proteins for healing and tissue re-
generation. Thromb Haemost 91: 4-15.
Anitua, E., M. Sanchez, A.T. Nurden, P. Nurden,
G. Orive, I. Andia (2006) New insights into
and novel applications for platelet-rich fibrin
therapies. Trends Biotechnol 24: 227-234.

Drengk/Zapf/Stürmer/Stürmer/Frosch 
Arpornmaeklong, P., M. Kochel, R. Depprich, N.R. Kubler, K.K. Wurzler (2004) Influence of platelet-rich plasma (PRP) on osteogenic differentiation of rat bone marrow stromal cells. An in vitro study. Int J Oral Maxillofac Surg 33: 60-70.

Bassleer, C., P. Gysen, J.M. Foidart, R. Bassleer, P. Franchimont (1986) Human chondrocytes in tridimensional culture. In Vitro Cell Dev Biol 22: 113-119.

Benya, P.D., S.R. Padilla, M.E. Nimni (1978) Independent regulation of collagen types by chondrocytes during the loss of differentiated function in culture. Cell 15: 1313-1321.

Binette, F., D.P. McQuaid, D.R. Haudenschild, P.C. Yaeger, J.M. McPherson, R. Tubo (1998) Expression of a stable articular cartilage phenotype without evidence of hypertrophy by adult human articular chondrocytes in vitro. J Orthop Res 16: 207-216.

Blanco, F.J., Y. Geng, M. Lotz (1995) Differentiation-dependent effects of IL-1 and TGF-beta on human articular chondrocyte proliferation are related to inducible nitric oxide synthase expression. J Immunol 154: 40184026.

Bosnakovski, D., M. Mizuno, G. Kim, T. Ishiguro, M. Okumura, T. Iwanaga, T. Kadosawa, T. Fujinaga (2004) Chondrogenic differentiation of bovine bone marrow mesenchymal stem cells in pellet cultural system. Exp Hematol 32: 502-509.

-Buckwalter, J.A., H.J. Mankin (1998) Articular cartilage: tissue design and chondrocytematrix interactions. Instr Course Lect 47: 477-486.

Celotti, F., A. Colciago, P. Negri-Cesi, A. Pravettoni, R. Zaninetti, M.C. Sacchi (2006) Effect of platelet-rich plasma on migration and proliferation of SaOS-2 osteoblasts: role of platelet-derived growth factor and transforming growth factor-beta. Wound Repair Regen 14: 195-202.

Choi, B.H., S.J. Zhu, B.Y. Kim, J.Y. Huh, S.H. Lee, J.H. Jung (2005) Effect of platelet-rich plas$\mathrm{ma}(\mathrm{PRP})$ concentration on the viability and proliferation of alveolar bone cells: an in vitro study. Int J Oral Maxillofac Surg 34: 420424.

Dugrillon, A., H. Eichler, S. Kern, H. Kluter (2002) Autologous concentrated plateletrich plasma (cPRP) for local application in bone regeneration. Int J Oral Maxillofac Surg 31: 615-619.

Farrag, T.Y., M. Lehar, P. Verhaegen, K.A. Carson, P.J. Byrne (2007) Effect of platelet rich plasma and fibrin sealant on facial nerve regeneration in a rat model. Laryngoscope 117: 157-165.

Fennis, J.P., P.J. Stoelinga, J.A. Jansen (2004) Mandibular reconstruction: a histological and histomorphometric study on the use of autogenous scaffolds, particulate corticocancellous bone grafts and platelet rich plasma in goats. Int J Oral Maxillofac Surg 33: 48-55.
Ferreira, C.F., M.C. Carriel Gomes, J.S. Filho, J.M. Granjeiro, C.M. Oliveira Simoes, S Magini Rde (2005) Platelet-rich plasma influence on human osteoblasts growth. Clin Oral Implants Res 16: 456-460.

Fortier, L.A., A.J. Nixon, J. Williams, C.S. Cable (1998) Isolation and chondrocytic differentiation of equine bone marrow-derived mesenchymal stem cells. Am J Vet Res 59: 1182 1187.

Fritz, J., C. Gaissmaier, B. Schewe, K. Weise (2006) Biologische Knorpelrekonstruktion im Kniegelenk. Unfallchirurg 109: 563-576.

Frosch, K.H., I. Sondergeld, K. Dresing, T. Rudy, C.H. Lohmann, J. Rabba, D. Schild, J. Breme, K.M. Stuermer (2003) Autologous osteoblasts enhance osseointegration of porous titanium implants. J Orthop Res 21: 213 223.

Fuerst, G., R. Gruber, S. Tangl, F. Sanroman, G. Watzek (2003) Enhanced bone-to-implant contact by platelet-released growth factors in mandibular cortical bone: a histomorphometric study in minipigs. Int J Oral Maxillofac Implants 18: 685-690.

Gaissmaier, C., J. Fritz, T. Krackhardt, I. Flesch, W.K. Aicher, N. Ashammakhi (2005) Effect of human platelet supernatant on proliferation and matrix synthesis of human articular chondrocytes in monolayer and three-dimensional alginate cultures. Biomaterials 26: 1953-1960.

Gardner, D.L. (1994) Problems and paradigms in joint pathology. J Anat 184: 465-476.

Gray, A.J., J.E. Bishop, J.T. Reeves, G.J. Laurent (1993) A alpha and B beta chains of fibrinogen stimulate proliferation of human fibroblasts. J Cell Sci 104: 409-413.

Graziani, F., S. Ivanovski, S. Cei, F. Ducci, M Tonetti, M. Gabriele (2006) The in vitro effect of different PRP concentrations on osteoblasts and fibroblasts. Clin Oral Implants Res 17: 212-219.

Gruber, R., F. Karreth, B. Kandler, G. Fuerst, A. Rot, M.B. Fischer, G. Watzek (2004) Plateletreleased supernatants increase migration and proliferation, and decrease osteogenic differentiation of bone marrow-derived mesenchymal progenitor cells under in vitro conditions. Platelets 15: 29-35.

Gruber, R., F. Varga, M.B. Fischer, G. Watzek (2002) Platelets stimulate proliferation of bone cells: involvement of platelet-derived growth factor, microparticles and membranes. Clin Oral Implants Res 13: 529-535.

Guerne, P.A., F. Blanco, A. Kaelin, A. Desgeorges, M. Lotz (1995) Growth factor responsiveness of human articular chondrocytes in aging and development. Arthritis Rheum 38: 960-968.

Johnson, L.L. (2001) Arthroscopic abrasion arthroplasty: a review. Clin Orthop Relat Res 391(suppl): S306-S317.
Kaps, C., A. Loch, A. Haisch, H. Smolian, G.R. Burmester, T. Haupl, M. Sittinger (2002) Human platelet supernatant promotes proliferation but not differentiation of articular chondrocytes. Med Biol Eng Comput 40: 485-490.

Kim, H.K., M.E. Moran, R.B. Salter (1991) The potential for regeneration of articular cartilage in defects created by chondral shaving and subchondral abrasion. An experimental investigation in rabbits. J Bone Joint Surg Am 73: 1301-1315.

Kitoh, H., T. Kitakoji, H. Tsuchiya, H. Mitsuyama, H. Nakamura, M. Katoh, N. Ishiguro (2004) Transplantation of marrowderived mesenchymal stem cells and platelet-rich plasma during distraction osteogenesis - a preliminary result of three cases. Bone 35: 892-898.

Kuettner, K.E., V.A. Memoli, B.U. Pauli, N.C. Wrobel, E.J. Thonar, J.C. Daniel (1982) Synthesis of cartilage matrix by mammalian chondrocytes in vitro. II. Maintenance of collagen and proteoglycan phenotype. J Cell Biol 93: 751-757.

Lanas, A., P. Haggerty, B.I. Hirschowitz (1994) Ingestion of aspirin prevents platelet-induced human fibroblast growth. Implications for peptic ulcer healing. Scand J Gastroenterol 29: 17-22.

Dindeboom, J.A., K.R. Mathura, I.H. Aartman, F.H. Kroon, D.M. Milstein, C. Ince (2007) Influence of the application of platelet-enriched plasma in oral mucosal wound healing. Clin Oral Implants Res 18: 133-139.

-Magnuson, P.B (1974) The classic: joint debridement: surgical treatment of degenerative arthritis. Clin Orthop Relat Res 101: 4-12.

Majumdar, M.K., V. Banks, D.P. Peluso, E.A. Morris (2000) Isolation, characterization, and chondrogenic potential of human bone marrow-derived multipotential stromal cells. J Cell Physiol 185: 98-106.

Marlovits, S., M. Hombauer, M. Truppe, V. Vecsei, W. Schlegel (2004) Changes in the ratio of type-I and type-II collagen expression during monolayer culture of human chondrocytes. J Bone Joint Surg Br 86: 286-295.

-Marx, R.E., E.R. Carlson, R.M. Eichstaedt, S.R. Schimmele, J.E. Strauss, K.R. Georgeff (1998) Platelet-rich plasma: growth factor enhancement for bone grafts. Oral Surg Oral Med Oral Pathol Oral Radiol Endod 85: 638646.

Murray, M.M., B. Forsythe, F. Chen, S.J. Lee, J.J. Yoo, A. Atala, A. Steinert (2006) The effect of thrombin on ACL fibroblast interactions with collagen hydrogels. J Orthop Res 24: 508-515.

Niethard, F.U., U. Schneider (2004) Cartilage is sturdier - progress in autologous chondrocyte transplantation (in German). Z Orthop Ihre Grenzgeb 142: 527-528. 
Pittenger, M.F., A.M. Mackay, S.C. Beck, R.K. Jaiswal, R. Douglas, J.D. Mosca, M.A. Moorman, D.W. Simonetti, S. Craig, D.R. Marshak (1999) Multilineage potential of adult human mesenchymal stem cells. Science 284 : 143-147.

Pridie, K. (1959) A method of resurfacing osteoarthritic knee joints. J Bone Joint Surg Br 41: 618-619.

-Sanchez, M., J. Azofra, E. Anitua, I. Andia, S. Padilla, J. Santisteban, I. Mujika (2003) Plasma rich in growth factors to treat an articular cartilage avulsion: a case report. Med Sci Sports Exerc 35: 1648-1652.

-Steadman, J.R., W.G. Rodkey, J.J. Rodrigo (2001) Microfracture: surgical technique and rehabilitation to treat chondral defects. Clin Orthop Relat Res 391(suppl): S362-S369.

Tallheden, T., C. Karlsson, A. Brunner, J. Van Der Lee, R. Hagg, R. Tommasini, A. Lindahl (2004) Gene expression during redifferentiation of human articular chondrocytes. Osteoarthritis Cartilage 12: 525-535. van der Kraan, P., E. Vitters, W. van den Berg (1992) Differential effect of transforming growth factor beta on freshly isolated and cultured articular chondrocytes. J Rheumatol 19: 140-145.

Vogel, J.P., K. Szalay, F. Geiger, M. Kramer, W. Richter, P. Kasten (2006) Platelet-rich plasma improves expansion of human mesenchymal stem cells and retains differentiation capacity and in vivo bone formation in calcium phosphate ceramics. Platelets 17: 462-469.

von der Mark, K., V. Gauss, H. von der Mark, P. Muller (1977) Relationship between cell shape and type of collagen synthesised as chondrocytes lose their cartilage phenotype in culture. Nature 267: 531-532.

-Weibrich, G., S.H. Gnoth, M. Otto, T.E. Reichert, W. Wagner (2002) Growth stimulation of human osteoblast-like cells by thrombocyte concentrates in vitro (in German). Mund Kiefer Gesichtschir 6: 168-174.
Weibrich, G., W.K. Kleis, M. Kunz-Kostomanolakis, A.H. Loos, W. Wagner (2001) Correlation of platelet concentration in platelet-rich plasma to the extraction method, age, sex, and platelet count of the donor. Int J Oral Maxillofac Implants 16: 693-699.

Weiser, L., M. Bhargava, E. Attia, P.A. Torzilli (1999) Effect of serum and platelet-derived growth factor on chondrocytes grown in collagen gels. Tissue Eng 5: 533-544.

Worster, A.A., A.J. Nixon, B.D. Brower-Toland, J. Williams (2000) Effect of transforming growth factor $\beta 1$ on chondrogenic differentiation of cultured equine mesenchymal stem cells. Am J Vet Res 61: 1003-1010.

Yazawa, M., H. Ogata, A. Kimura, T. Nakajima, T. Mori, N. Watanabe (2004) Basic studies on the bone formation ability by platelet rich plasma in rabbits. J Craniofac Surg 15: 439446.

Zangi, L., R. Rivkin, I. Kassis, L. Levdansky, G. Marx, R. Gorodetsky (2006) High-yield isolation, expansion, and differentiation of rat bone marrow-derived mesenchymal stem cells with fibrin microbeads. Tissue Eng 12: 2343-2354. 